\title{
CALAGEM EM SISTEMA DE SEMEADURA DIRETA E EFEITOS SOBRE A ACIDEZ DO SOLO, DISPONIBILIDADE DE NUTRIENTES E PRODUTIVIDADE DE MILHO E SOJ A ${ }^{(1)}$
}

\author{
S. G. MOREIRA(2), J . C. KIEHL (3), L. I.PROCHNOW(3) \& V.PAULETTI ${ }^{(4)}$
}

\begin{abstract}
RESUMO
Estudou-se o efei to da calagem na correção da acidez do solo, disponibi lidade de alguns nutrientes e produtividade de milho e soja em um Latossolo Vermelho com diferentes tempos de cultivo sob sistema de semeadura direta (três, seis e nove anos). Quatro doses de calcário $(0,33,3,66,7$ e $100 \%$ da quantidade calculada para elevar a saturação por bases a $70 \%$ ) foram aplicadas na superfície, além de um tratamento adicional, constituído pela maior dose, incorporada na camada de 0-20 cm. Houve distribuição mais uniforme em profundidade de $\mathrm{Ca}, \mathrm{Mg}$ e V\% no solo com o maior tempo de cultivo. Nesse solo, os teores de Al foram baixos e não variaram com a profundidade, enquanto os valores de pH variaram pouco no perfil. No solo com três anos de cultivo, a maior dose de calcário aplicada na superfície resultou em maiores teores de Ca e Mg na camada de 0-5 cm, enquanto o pH e a V\% não variaram. A incorporação da dose integral elevou o pH e o teor de Ca na camada de $10-20 \mathrm{~cm}$ e o teor de $\mathrm{Mg}$ e a V\% das camadas de 10-20 e $20-30 \mathrm{~cm}$, diminuindo o teor de Al da camada de $20-30 \mathrm{~cm}$. No solo com seis anos de cultivo, pH, Ca e Mg da camada superficial geralmente aumentaram com a aplicação das maiores doses na superfície. A incorporação, em geral, diminuiu o teor de Al e aumentou os de Ca e Mg e a V\% nas camadas inferiores do solo. No solo com maior tempo de cultivo, a aplicação superficial quase sempre acarretou maiores teores de Ca e Mg e maiores valores de $\mathrm{pH}$ e V\% na camada de 0-5 cm do que a incorporação. Os teores de N, P, K e S nas folhas de soja não variaram com a calagem. As produções de grãos de milho e soja não foram influenciadas pela calagem, mas o local com seis anos apresentou a maior produtividade de grãos de soja.
\end{abstract}

Termos de indexação: cálcio, magnésio, fósforo, potássio, enxofre.

(1) Parte da Tese de Mestrado do primeiro autor. Recebido para publicação em dezembro de 1999 e aprovado em outubro de 2000.

(2) Doutorando do Curso de Solos e Nutrição de Plantas, Escola Superior de Agricultura Luiz de Queiroz - ESALQ/USP. Av. Pádua Dias, 11, Caixa Postal 9, CEP 13418-900 Piracicaba (SP). E-mail: sgmoreir@carpa.ciagri.usp.br

(3) Professor do Departamento de Solos e Nutrição de Plantas, ESALQ/USP

(4) Pesquisador da Fundação ABC, BR 151, Km 155,5, Caixa Postal 1003, CEP 84166-900 Castro (PR). 


\title{
SUMMARY: LIMING UNDER NO-TILLAGE AND EFFECTS ON SOIL ACIDITY, SOIL NUTRIENT AVAILABILITY AND CORN AND SOYBEAN YIELD
}

\begin{abstract}
A fiel d experiment was conducted in thestateof Paraná, Brazil, to evaluatetheeffect of liming on soil acidity, soil nutrient availability and yields of maizeand soybeans on a Red Latosol (Oxysol), which had been cultivated for thre, six and nine years under no-tillage (NT). Lime was broadcasted to the soil surface in four rates- 0, 33.3, 66.7 and $100 \%$ of the amount calculated to increasethesoil basesaturation (V\%) to $70 \%$; an additional treatment consisted of broadcasting the highest rate of lime and mixing it to the $0-20 \mathrm{~cm}$ layer of the soil. Calcium, magnesium and V\% showed the most uniform distribution in the profile when thesoil was cultivated under NT for nineyears; in addition, exchangeableAl was low and $\mathrm{pH}$ was constant in all thestudied soil layers. In thesoil cultivated for threeyears, the highest figures for $\mathrm{Ca}$ and $\mathrm{Mg}$ in the $0-5 \mathrm{~cm}$ layer wereobserved when li mewas broadcasted on soil surfaceat full rate, whilepH and $\mathrm{V} \%$ did not changewith liming. Thehighest rateof limemixed in the soil increased $\mathrm{pH}$ and $\mathrm{Ca}$ content of the $10-20 \mathrm{~cm}$ layer, as well as the Mg and $\mathrm{V} \%$ of both layers of $10-20$ and $20-30 \mathrm{~cm}$. This treatment al so reduced the Al content of the 20-30 cm layer. In the soil with six-year cultivation period pH, Ca and $\mathrm{Mg}$ of the upper layer generally increased with thehighest rates of li mebroadcasted to thesoil surface. When mixed to thesoil, limereduced theexchangeableAl and increased the $\% \%$ and thecontent of $\mathrm{Ca}$ and $\mathrm{Mg}$ of the deepest soil layers. In the soil cultivated for nine years, lime influenced only the surfacelayer; when applied on the surface, the increase in $\mathrm{pH}, \mathrm{V} \%, \mathrm{Ca}$ and $\mathrm{Mg}$ by limewas generally higher than when mixed to thesoil. Liming did not influencethecontents of $\mathrm{N}, \mathrm{P}, \mathrm{K}$ and $\mathrm{S}$ in the soybean leaves. Maizeand soybean grain yied ds were not influenced by liming, but the soil with six years of cultivation under NT produced thehighest soybean yield.
\end{abstract}

Index terms: calcium, magnesium, nitrogen, phosphorus, potassium, sulphur.

\section{INTRODUÇÃO}

Coma adoção do sistema de semeadura direta (SSD) no Brasil, com o qual muitos agricultores conseguiram melhorar os atributos do solo e aumentar a produtividade das culturas, tornou-se necessário o estudo de métodos alternativos para a correção da acidez que não exigissem a incorporação do corretivo (Pottker \& Ben, 1998). Uma das principais dúvidas relacionava-secom a possibilidade de manter a produtividade das culturas em SSD, em áreas onde a correção da acidez havia sido adequadamente realizada antes da adoção do sistema e em áreas onde este procedimento não havia sido inicial mente adotado, tendo si do aplicado o cal cário apenas na superfície do solo (Sá, 1995).

Com a finalidade de responder a tais questionamentos, trabal hos vêm sendo realizados, com resultados satisfatórios (Sá, 1995; Santos et al., 1995; Oliveira \& Pavan, 1996). Sá (1995) não encontrou diferenças nas produtividades de soja, trigo e milho quanto às diferentes formas de aplicação do corretivo em solos ácidos do Paraná, cultivados por longo tempo sob o SSD. Nesses trabal hos, embora os cultivares suscetíveis à acidez mostrassem inicial mente respostas à incorporação, foi notória a correção do solo pela ação do cal cário aplicado em superfície. O mel hor retorno econômico foi obtido quando o corretivo foi aplicado na superfície.

Atualmente, não têm sido obtidos aumentos na produtividade de grãos com a aplicação de calagem superficial em sol os ácidos brasileiros cultivados há longo tempo sobSSD (Caires et al., 1998; Caires et al., 1999). Assim, existem especulações de que, à medi da que aumenta o tempo de cultivo dos solos com a semeadura direta, as doses de calcário possam ser diminuídas, cujas razões para isso precisam ser mais bem estudadas. Além disso, existem dúvidas quanto à dose a ser empregada, de acordo com o tempo de adoção do SSD.

No Brasil, poucos estudos têm reportado o efeito da calagem nos teores de nutrientes nas fol has e seus reflexos na produção das culturas, sendo, em alguns casos, contraditórios. Em estudo realizado durante quatro anos por Oliveira \& Pavan (1996), os teores de $\mathrm{Ca}$ e $\mathrm{Mg}$ nas fol has de soja não foram alterados com a aplicação do cal cário na superfície do solo, com exceção do último ano, quando os teores deCa diminuíram. Durante dois anos deaval iações, Caires et al. (1998) não observaram variações nas concentrações de N, P, K, S, Ca e Mg nas fol has de soja com a aplicação de doses de calcário 
superficialmente. Por outro lado, em outro estudo, conduzido em solo mantido durante 15 anos sob SSD, foi obtido aumento da concentração de Ca nas fol has de soja e de Mg nas fol has de soja e trigo (Caires et al., 1999). Nesse estudo, observou-se que os teores de Ca e Mg nas fol has de milho e os de $\mathrm{S}$ nas fol has de milho, soja e trigo não foram modificados com a calagem superficial.

Diantedo exposto, realizou-se o presente trabalho com o objetivo de aval iar o efeito do cal cário, aplicado em diferentes doses na superfície e incorporado ao sol o na camada de 0-20 cm de profundidade, sobre a acidez do solo, disponibilidade de nutrientes às plantas e produtividades de soja e milho cultivados em um Latossol o Vermel ho submetido a diferentes tempos de cultivo sob o sistema de semeadura direta.

\section{MATE RIAL E MÉTODOS}

O trabalho foi desenvolvido em condições de campo, em um Latossolo Vermel ho argiloso submetido a diferentes tempos de cultivo sob o sistema de semeadura direta (três, seis enoveanos), no município de Tibagi, PR. Os locais com três e seis anos de cultivo pertenciam à Fazenda Lagoa e à Fazenda F ortaleza, respectivamente, sendo áreas de lavouras de grãos; o local com nove anos de cultivo situava-se em área experimental da Fundação ABC.

Antes da adoção do SSD nesses locais, o sol o foi preparado com arado de aiveca $(0-20 \mathrm{~cm})$ e a acidez foi corrigida com calcário dolomítico (PRNT $=84$, $\mathrm{PN}=98, \mathrm{RE}=89, \mathrm{CaO}=27$ e $\mathrm{MgO}=20 \%$ ), incorporando-se o corretivo na camada de $0-20 \mathrm{~cm}$ com duas passadas degrade niveladora, empregandose a dose necessária para elevar a saturação por bases a $70 \%$. Calagens posteriores foram efetuadas sempre que necessário, aplicando-se o calcário superficialmente. Assim, o solo com nove anos de cultivo recebeu duas aplicações de cal cário de $2 \mathrm{t}$ ha-1 cada uma, o solo com seis anos em SSD, uma aplicação de $2 \mathrm{t} \mathrm{ha} \mathrm{a}^{-1}$, enquanto o solo com três anos de cultivo não recebeu aplicação do corretivo após a calagem inicial. Na instal ação do experimento (maio de 1998), os sol os não recebiam aplicação de cal cário há três anos.
O delineamento experimental empregado foi o de blocos ao acaso, com tratamentos em arranjo fatorial $3 \times 5$ (3 tempos de cultivo x 5 doses de cal cário), com parcelas subsubdi vididas, com quatro repetições. As parcelas principais constituíram-se do fatorial tempos de cultivo $x$ doses de calcário, totalizando 60 parcelas; as subparcelas foram formadas pelas duas épocas de amostragem, efetuadas após aplicação do calcário, e as subsubparcelas, pelas camadas de solo amostradas (0-5, 5-10, 10-20 e 20-30 cm). As parcelas eram de $6,4 \times 7 \mathrm{~m}$, nos tratamentos com três e seis anos de cultivo, ede $8,30 \times 12,50 \mathrm{~m}$, notratamento com nove anos de SSD.

O fator dose correspondeu a quatro doses de cal cário dol omítico, aplicadas na superfície do solo: $0,33,3,66,7$ e $100 \%$ da quantidade necessária para elevar a saturação por bases a $70 \%$, e de um tratamento adicional, empregando $100 \%$ da dose de calcárionecessária para elevar a saturação por bases a $70 \%$, incorporando-se, porém, o corretivo na camada de 0-20 cm. As doses necessárias para elevar a saturação por bases a 70\% foram de 3.030, 3.380 e $2.485 \mathrm{~kg} \mathrm{ha}^{-1}$, respectivamente, nos locais com três, seis e nove anos sob SSD. O cal cário foi distribuído manualmente na superfície do solo e, nas parcelas onde foi incorporado, com arado de discos. Para determinar as doses de cal cário, foi efetuada a coleta de 20 amostras simples de terra na profundidade de $0-20 \mathrm{~cm}$, em cada tratamento (três, seis e nove anos de cultivo), que, depois, foram reunidas em uma amostra composta por tratamento. Os resultados das anál ises químicas dessas amostras encontram-seno quadro 1.

Após a calagem, em maio de 1998, efetuou-se a semeadura de aveia branca, nos tratamentos com seis e nove anos de cultivo, e de aveia preta, no de três anos de cultivo, com o objetivo de produzir cobertura morta para o solo. A semeadura, sem adubo, foi realizada mecanicamente, com semeadora de parcelas regulada para distribuir 60 e $70 \mathrm{~kg} \mathrm{ha}^{-1}$ de sementedeaveia preta ebranca, respectivamente, no espaçamento de $15 \mathrm{~cm}$ entre as linhas.

A soja (Glycine max cV. EMBRAPA 59) foi semeada em outubro/1998, com espaçamento de $45 \mathrm{~cm}$ entrelinhas e 16 plantas por metro linear, e

Quadro 1. Propriedades quími cas do Latossolo Vermelho na camada de 0-20 cm antes da calagem inicial, para diferentes tempos de cultivo sob sistema de semeadura direta (TCSSD)

\begin{tabular}{|c|c|c|c|c|c|c|c|c|c|c|c|c|}
\hline TCSSD & $\mathrm{pH}_{\mathrm{CaCl}_{2}}$ & Presina & MO & $\mathbf{H}+\mathbf{A l}$ & Al & $\mathbf{K}$ & $\mathrm{Ca}$ & Mg & SB & СТC & $\mathbf{v}$ & $\mathbf{m}$ \\
\hline ano & \multicolumn{3}{|c|}{$-m g d m^{-3}-$} & \multicolumn{7}{|c|}{$-\mathrm{mmol}_{\mathrm{c}} \mathrm{dm}^{-3}$} & \multicolumn{2}{|c|}{$-\%$} \\
\hline Três & 5,0 & 33 & 55 & 58 & 1 & 3,7 & 28 & 26 & 57,7 & 115,7 & 50 & 1,7 \\
\hline Seis & 4,8 & 41 & 47 & 72 & 2 & 6,7 & 47 & 18 & 71,7 & 143,7 & 50 & 2,7 \\
\hline Nove & 5,1 & 44 & 47 & 52 & 0 & 2,6 & 34 & 21 & 57,6 & 109,6 & 53 & 0,0 \\
\hline
\end{tabular}


adubada com $200 \mathrm{~kg} \mathrm{ha}^{-1}$ da fórmula 0-20-20. As sementes de soja foram inoculadas com estirpes selecionadas de Bradyrhizobium, em meio líquido com $64 \mathrm{~g}$ de molibdato de sódio para $50 \mathrm{~kg}$ de sementes, e depois tratadas com $150 \mathrm{~g}$ i.a. dethiram para cada $50 \mathrm{~kg}$ de sementes.

Após a colheita da soja (maio de 1999), quando os solos com três, seis e nove anos sob SSD estavam com quatro, sete e dez anos sob SSD, efetuou-se a semeadura deaveia preta em todos os locais, utilizando os mesmos procedimentos do ano anterior. Em outubro/1999, o milho (Zea mays cv. XL8392) foi semeado com espaçamento de $80 \mathrm{~cm}$ entrelinhas e cinco plantas por metro linear e adubado com $300 \mathrm{~kg} \mathrm{ha}^{-1}$ da fórmula $10-20-20+1 \%$ de Zn.

O efeito das doses de cal cário na disponibilidade de nutrientes foi avaliado pel o teor de nutrientes no sol o e nas fol has, durante o cultivo da soja, em duas épocas de amostragem. As coletas de terra foram realizadas no estádio V4 (três trifólios abertos) e no estádio R1 a R2, início do florescimento, respectivamente, nos dias 17/12/1998 e 26/01/1999, quando já havia chovido 1.174 e $1.305 \mathrm{~mm}$ após efetuada a calagem. Vale ressaltar que, após a semeadura da soja, houve uma estiagem de 37 dias (26/10 a 02/12/1998). Entrea semeadura ea colheita da soja choveu $767 \mathrm{~mm}$.

As amostras de terra foram coletadas nas profundidades de 0-5, 5-10, 10-20 e 20-30 cm, por meio de trado calador, em número de 10 amostras simples por parcela. As amostras foram secas em estufa a $40^{\circ} \mathrm{C}$, destorroadas, moídas e passadas em peneira de $2 \mathrm{~mm}$. As amostras de fol has foram coletadas em número de 20 trifólios por parcela, retirando-se a terceira folha a partir do ápice da planta. As fol has foram lavadas emágua desionizada, secas em estufa a $60^{\circ} \mathrm{C}$ até atingir massa constante e moídas. Nas amostras de terra, determinaram-se pH , Al, Ca e Mg, segundo método de Raij et al. (1987), sendo cal culados os val ores de saturação por bases. Nas amostras de fol ha, foram determinados os teores de N, P, K, S, Ca e Mg, conforme métodos descritos por Malavolta et al. (1997).

A col heita da soja foi feita manualmente no final de março/1999, e a do milho, em março/2000. Para a colheita da soja, a área útil de cada parcela correspondia às cinco linhas centrais, com quatro metros lineares por linha, e, para o milho, quatro linhas centrais, com dois metros lineares. Na col heita, utilizou-setrilha mecânica com debulhadora degrãos estacionária, tendo sido os grãos peneirados, pesados e submetidos à determinação de umidade em medidor universal. Para o cálculo das produtividades de milho e soja, considerou-se um teor de umidade nos grãos de $13 \%$.

Os resultados foram submetidos à análise de variância (teste $F$ ), e as médias comparadas pelo teste de Tukey a $5 \%$, para todos os atributos estudados. O teste demédias foi utilizado nolugar de regressões polinomiais, pelo fato de a forma de aplicação do calcárionão ter sido a mesma em todos os tratamentos.

Foram feitos estudos de correlaçãolinear simples entre os teores de macronutrientes ( $\mathrm{P}, \mathrm{K}, \mathrm{S}, \mathrm{Ca}$ e Mg) no solo e na folha, nas profundidades de 0-5, 5-10, 10-20 e 20-30 cm, separando-se o tratamento com incorporação do corretivo dos tratamentos sem revolvimento do solo, dentro de cada época de amostragem.

\section{RESULTADOS E DISCUSSÃO}

Houve interação tripla de doses de calcário, tempos de cultivo sob o sistema de semeadura dir eta e profundidades de amostragem para todos os atributos do solo estudados (valores de $\mathrm{pH}$ e de saturação por bases, teores de Al, Ca e Mg). Como não houve interação de quarto grau, incluindo as épocas de amostragem, os resultados apresentados são referentes às médias das duas épocas de amostragem.

No sol o com três anos sob SSD, não houve efeito das doses de calcário aplicadas superficialmente sobre os valores de $\mathrm{pH}$, nem quando o calcário foi incorporado, exceto na camada de $10-20 \mathrm{~cm}$, cujo pH se el evou com a aplicação das maiores quantidades do corretivo e quando este foi incorporado ao solo (Quadro 2). Santos et al. (1995) também não encontraram diferenças quanto ao pH do solo, entre a aplicação superficial e incorporada de $2 \mathrm{t} \mathrm{ha}^{-1}$ de cal cário (PRNT $=100 \%$ ) em um L atossolo Vermel hoEscuro, após três anos de cultivos sob SSD. Em condições de cl ima temperado, Hargrove et al . (1982) não verificaram diferenças no $\mathrm{pH}$ da camada superficial do solo devidas aos sistemas de cultivo, enquanto Kitur et al. (1994) observaram maior val or no SSD do que no sistema de semeadura convencional (SSC).

Na camada superficial do solo com seis anos sob SSD, a aplicação de $100 \%$ da dose de calcário na superfície propiciou um val or de $\mathrm{pH}(5,51)$ superior ao obtido com a aplicação da mesma dose de forma incorporada $(4,76)$. Na camada de $20-30 \mathrm{~cm}$, por sua vez, ocorreu o inverso. No solo com nove anos sob SSD, a aplicação superficial de $2 / 3$ da dose resultou em maior pH $(5,46)$ do que a aplicação da dose total incorporada $(5,03)$. Esses resultados eram esperados, uma vez que o revolvimento do solo provoca a mistura do cal cário com uma camada mais espessa do perfil do solo.

Quando o cal cárioécolocado na superfície, menor volume de solo é atingido, concentrando seu efeito inicialmente nas camadas superficiais. N o entanto, segundo alguns autores, com o aumento do tempo de cultivo em SSD, os valores de $\mathrm{pH}$ da camada subsuperficial tendem a aumentar. Oliveira \& Pavan (1996) obtiveram aumento de $\mathrm{pH}$ até $40 \mathrm{~cm}$ de 
Quadro 2. Valores de pH, Ca, Mg, Al trocável e saturação por bases do Latosssolo Vermelho, considerando as doses de calcário, para diferentes tempos de cultivo sob sistema de semeadura direta (TCSSD) e profundidades

\begin{tabular}{|c|c|c|c|c|c|}
\hline \multirow{2}{*}{ Profundidade } & \multicolumn{5}{|c|}{ Dose de calcário(1) } \\
\hline & $100 I$ & $100 \mathrm{~S}$ & $66,7 \mathrm{~S}$ & $33,3 \mathrm{~S}$ & $\mathbf{0}$ \\
\hline $\mathrm{cm}$ & \multicolumn{5}{|c|}{ pH } \\
\hline $\begin{array}{c}0-5 \\
5-10 \\
10-20 \\
20-30\end{array}$ & $\begin{array}{l}5,45 \mathrm{aA}(2) \\
5,25 \mathrm{abA} \\
5,50 \mathrm{aA} \\
4,90 \mathrm{bA}\end{array}$ & $\begin{array}{l}5,61 \text { aA } \\
5,36 \text { aA } \\
5,31 \text { aAB } \\
4,69 \mathrm{bA}\end{array}$ & $\begin{array}{l}\text { Três anos } \\
5,50 \mathrm{aA} \\
5,46 \mathrm{aA} \\
5,18 \mathrm{aABC} \\
4,74 \mathrm{bA}\end{array}$ & $\begin{array}{l}5,48 \mathrm{aA} \\
5,33 \mathrm{abA} \\
4,94 \mathrm{bcBC} \\
4,56 \mathrm{cA}\end{array}$ & $\begin{array}{l}5,46 \mathrm{aA} \\
5,31 \mathrm{aA} \\
4,83 \mathrm{bC} \\
4,55 \mathrm{bA}\end{array}$ \\
\hline $\begin{array}{c}0-5 \\
5-10 \\
10-20 \\
20-30\end{array}$ & $\begin{array}{l}4,76 \mathrm{aB} \\
4,85 \mathrm{aA} \\
4,85 \mathrm{aA} \\
4,63 \mathrm{aA}\end{array}$ & $\begin{array}{l}5,51 \mathrm{aA} \\
4,66 \mathrm{bA} \\
4,51 \mathrm{bcA} \\
4,21 \mathrm{cB}\end{array}$ & $\begin{array}{c}\text { Seis anos } \\
5,13 \mathrm{aAB} \\
4,60 \mathrm{bA} \\
4,50 \mathrm{bA} \\
4,20 \mathrm{bB}\end{array}$ & $\begin{array}{l}5,16 \mathrm{aAB} \\
4,70 \mathrm{bA} \\
4,66 \mathrm{bcA} \\
4,25 \mathrm{CAB}\end{array}$ & $\begin{array}{l}5,08 \mathrm{aB} \\
4,66 \mathrm{bA} \\
4,49 \mathrm{bcA} \\
4,24 \mathrm{cAB}\end{array}$ \\
\hline $\begin{array}{c}0-5 \\
5-10 \\
10-20 \\
20-30\end{array}$ & $\begin{array}{l}5,03 \mathrm{aB} \\
4,98 \mathrm{aA} \\
5,15 \mathrm{aA} \\
4,90 \mathrm{aA}\end{array}$ & $\begin{array}{l}5,40 \mathrm{aAB} \\
5,06 \mathrm{aA} \\
5,14 \mathrm{aA} \\
5,04 \mathrm{aA}\end{array}$ & $\begin{array}{c}\text { Nove anos } \\
5,46 \mathrm{aA} \\
4,89 \mathrm{bA} \\
5,09 \mathrm{abA} \\
4,96 \mathrm{bA}\end{array}$ & $\begin{array}{l}5,33 \text { aAB } \\
4,93 \text { abA } \\
4,93 \text { abA } \\
4,86 \mathrm{bA}\end{array}$ & $\begin{array}{l}5,04 \mathrm{aB} \\
4,71 \mathrm{aA} \\
4,85 \mathrm{aA} \\
4,84 \mathrm{aA}\end{array}$ \\
\hline \multirow[t]{3}{*}{ C.V. (\%) } & 4,8 & & & & \\
\hline & \multicolumn{5}{|c|}{$\mathrm{Ca}$} \\
\hline & \multicolumn{5}{|c|}{$-\mathrm{mmol}_{\mathrm{c}} \mathrm{dm}^{-3}$} \\
\hline $\begin{array}{c}0-5 \\
5-10 \\
10-20 \\
20-30\end{array}$ & $\begin{array}{l}64 \mathrm{aB} \\
62 \mathrm{aA} \\
50 \mathrm{aA} \\
28 \mathrm{bA}\end{array}$ & $\begin{array}{l}88 \text { aA } \\
60 \mathrm{bA} \\
43 \mathrm{bcAB} \\
23 \mathrm{cA}\end{array}$ & $\begin{array}{c}\text { Três anos } \\
73 \mathrm{aAB} \\
57 \mathrm{aA} \\
33 \mathrm{bcAB} \\
20 \mathrm{cA}\end{array}$ & $\begin{array}{l}67 \mathrm{aB} \\
52 \mathrm{aA} \\
32 \mathrm{bB} \\
16 \mathrm{bA}\end{array}$ & $\begin{array}{l}64 \mathrm{aB} \\
51 \mathrm{aA} \\
31 \mathrm{bcB} \\
16 \mathrm{cA}\end{array}$ \\
\hline $\begin{array}{c}0-5 \\
5-10 \\
10-20 \\
20-30\end{array}$ & $\begin{array}{l}53 a C \\
50 a A \\
56 a A \\
40 a A\end{array}$ & $\begin{array}{l}91 \mathrm{aA} \\
47 \mathrm{bA} \\
33 \mathrm{bcB} \\
21 \mathrm{cB}\end{array}$ & $\begin{array}{c}\text { Seis anos } \\
88 \mathrm{aA} \\
46 \mathrm{bA} \\
33 \mathrm{bcB} \\
19 \mathrm{cB}\end{array}$ & $\begin{array}{l}76 \mathrm{aAB} \\
48 \mathrm{bA} \\
43 \mathrm{bAB} \\
23 \mathrm{CAB}\end{array}$ & $\begin{array}{l}69 \mathrm{aBC} \\
46 \mathrm{bA} \\
35 \mathrm{bcB} \\
20 \mathrm{cB}\end{array}$ \\
\hline $\begin{array}{c}0-5 \\
5-10 \\
10-20 \\
20-30\end{array}$ & $\begin{array}{l}50 \mathrm{aB} \\
49 \mathrm{aA} \\
51 \mathrm{aA} \\
37 \mathrm{aA}\end{array}$ & $\begin{array}{l}81 \mathrm{aA} \\
52 \mathrm{bA} \\
49 \mathrm{bA} \\
46 \mathrm{bA}\end{array}$ & $\begin{array}{c}\text { Nove anos } \\
84 \mathrm{aA} \\
48 \mathrm{bA} \\
45 \mathrm{bA} \\
42 \mathrm{bA}\end{array}$ & $\begin{array}{l}73 \mathrm{aA} \\
45 \mathrm{bA} \\
46 \mathrm{bA} \\
36 \mathrm{bA}\end{array}$ & $\begin{array}{l}55 \mathrm{aB} \\
40 \mathrm{aA} \\
44 \mathrm{aA} \\
39 \mathrm{aA}\end{array}$ \\
\hline \multirow[t]{2}{*}{ C.V. (\%) } & 20,9 & & & & \\
\hline & \multicolumn{5}{|c|}{ Mg } \\
\hline $\begin{array}{c}0-5 \\
5-10 \\
10-20 \\
20-30\end{array}$ & $\begin{array}{l}39 \mathrm{aC} \\
40 \mathrm{aA} \\
42 \mathrm{aA} \\
24 \mathrm{bA}\end{array}$ & $\begin{array}{l}66 \mathrm{aA} \\
48 \mathrm{bA} \\
33 \mathrm{cAB} \\
17 \mathrm{dA}\end{array}$ & $\begin{array}{c}\text { Três anos } \\
56 \mathrm{aAB} \\
41 \mathrm{bA} \\
27 \mathrm{cB} \\
13 \mathrm{dA}\end{array}$ & $\begin{array}{l}49 \mathrm{aBC} \\
40 \mathrm{abA} \\
27 \mathrm{bB} \\
12 \mathrm{cA}\end{array}$ & $\begin{array}{l}49 \mathrm{aBC} \\
40 \mathrm{aA} \\
26 \mathrm{bB} \\
11 \mathrm{cA}\end{array}$ \\
\hline $\begin{array}{c}0-5 \\
5-10 \\
10-20 \\
20-30\end{array}$ & $\begin{array}{l}32 \mathrm{aD} \\
33 \mathrm{aA} \\
37 \mathrm{aA} \\
27 \mathrm{aA}\end{array}$ & $\begin{array}{l}64 \mathrm{aA} \\
28 \mathrm{bA} \\
18 \mathrm{bcB} \\
11 \mathrm{cB}\end{array}$ & $\begin{array}{c}\text { Seis anos } \\
54 \mathrm{aAB} \\
27 \mathrm{bA} \\
18 \mathrm{bcB} \\
11 \mathrm{cB}\end{array}$ & $\begin{array}{l}50 \mathrm{aBC} \\
28 \mathrm{bA} \\
25 \mathrm{bcAB} \\
14 \mathrm{CAB}\end{array}$ & $\begin{array}{l}39 \mathrm{aCD} \\
27 \mathrm{aA} \\
19 \mathrm{abB} \\
11 \mathrm{bB}\end{array}$ \\
\hline $\begin{array}{c}0-5 \\
5-10 \\
10-20 \\
20-30\end{array}$ & $\begin{array}{l}31 \mathrm{aB} \\
31 \mathrm{aA} \\
34 \mathrm{aA} \\
22 \mathrm{aA}\end{array}$ & $\begin{array}{l}47 \mathrm{aA} \\
33 \mathrm{bA} \\
27 \mathrm{bA} \\
26 \mathrm{bA}\end{array}$ & $\begin{array}{c}\text { Nove anos } \\
51 \mathrm{aA} \\
30 \mathrm{bA} \\
27 \mathrm{bA} \\
27 \mathrm{bA}\end{array}$ & $\begin{array}{l}48 \mathrm{aA} \\
30 \mathrm{bA} \\
25 \mathrm{bA} \\
25 \mathrm{bA}\end{array}$ & $\begin{array}{l}33 \mathrm{aB} \\
22 \mathrm{aA} \\
26 \mathrm{aA} \\
25 \mathrm{aA}\end{array}$ \\
\hline C.V. (\%) & 25,9 & & & & \\
\hline
\end{tabular}




\section{Quadro 2. Continuação}

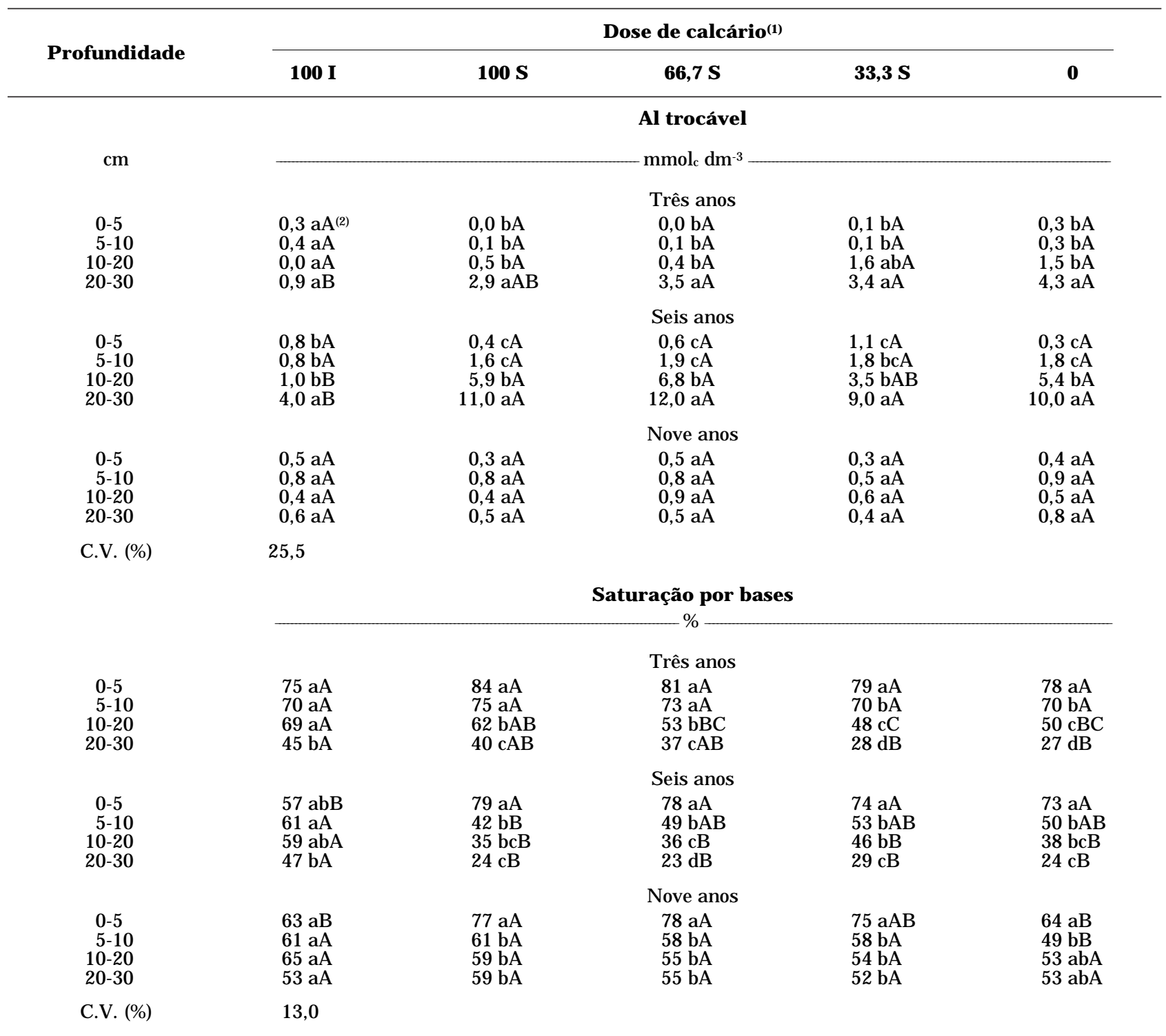

(1) Percentagem da dose necessária para el evar a saturação por bases a 70\%; I =incorporado a $20 \mathrm{~cm}$; S =aplicado superficialmente. (2) Análise de variância e teste de médias realizados com os dados transformados em $(\mathrm{Al}+1)^{1 / 2}$. Letras minúsculas comparam profundidades dentro de cada dose de cal cário em cada TCSSD e as maiúsculas comparam as doses dentro de cada TCSSD em cada profundidade, pelo teste de Tukey a $5 \%$.

profundidadeapós 32 meses da calagem superficial, em solos ácidos do Paraná. Resultados similares foram obtidos por Caires et al. (1998) e Caires et al. (1999) em solos de textura média.

No presente trabal ho, o tempo entre a aplicação do calcário (maio/1998) e a coleta das amostras (dez/1998 a jan/1999) não foi suficiente para que se efetuasse a correção das camadas mais profundas. Em sol os sob cl ima temperado, por outrolado, foram encontrados menores valores de $\mathrm{pH}$ na camada superficial de sol os sob SSD do que naqueles sob SSC
(Blevins et al ., 1986; Follett \& Peterson, 1988; Grove \& Blevins, 1988; Edwards et al., 1992; Franzluebbers \& Hons, 1996). Esse abaixamento de $\mathrm{pH}$ deveu-se, em muitos casos, ao efeito aci dificante das altas doses de N, empregadas principalmente nos monocultivos degramíneas.

No sol o com três anos sob SSD, houve efeito da dose máxima de calcário, quando incorporado, noteor de Al trocável apenas na camada de $20-30 \mathrm{~cm}$ (Quadro 2). É provável que a incorporação do corretivo na camada de 0-20 cm tenha facilitado sua 
movimentação no perfil do sol o e que o tempo entre a aplicação do corretivo e a retirada das amostras nãotenha sido suficiente para que ocorresse o efeito do calcário aplicado superficialmente. De forma semel hante, a calagem superficial não al terou o teor deAl trocável de um Latossolo Vermelho-Escuro há três anos sob SSD (Santos et al., 1995). O teor de Al de um solo canadense também não variou com a calagem (Arshad et al., 1997).

Nas camadas inferiores do solo com seis anos sob SSD, os teores de Al trocável apresentaram-se maiores que os das camadas superficiais e diminuíram com a incorporação do cal cário e, no solo com mai or tempo de cultivo, os teores não variaram com as doses de corretivo nem com as profundidades. Tem sido observada maior diminuição do Al nas camadas superficiais de solos onde o corretivo foi aplicado na superfície do que de solos onde esse material foi incorporado (Moschler et al., 1973; Sá, 1995), tendendo essa correção da acidez a seestender às camadas subsuperficiais ao longo do tempo de cultivo (Oliveira \& Pavan, 1996; Caires et al., 1998; Caires et al., 1999).

O efeito do calcário sobre a camada superficial do solo é maior quando o calcário é aplicado à superfície porque a incorporação o dilui em uma camada mais espessa de sol o. Decorrido certo tempo, embora seja um produto de baixa solubilidade, o calcário pode-se movimentar por meio de poros e canais formados por raízes mortas e organismos do solo (Sá, 1995; Oliveira \& Pavan, 1996), bem como por meio da formação de pares iônicos entre os produtos liberados pela decomposição dos restos culturais na superfície, com os íons $\mathrm{H}^{+}, \mathrm{Ca}^{2+} \mathrm{e} \mathrm{Mg}^{2+}$ presentes no solo, formando compostos com grande facilidade de movimentação no perfil. Com a lixiviação desses compostos, ocorre a reação dos pares iônicos com $\mathrm{H}^{+}$e $\mathrm{Al}^{3+}$ das camadas inferiores do solo, liberando $\mathrm{Ca}$ eMge, desta forma, aumentando o pH eos teores de Ca e Mg e diminuindo o Al tóxico (Miyazawa et al., 1996).

No sol o com menor tempo de cultivo, a maior dose de corretivo aplicada na superfície resultou em teores de Ca e Mg na camada de 0-5 cm maiores do que quando aplicada de forma incorporada (Quadro 2). $\mathrm{Na}$ camada superficial dos solos com os maiores tempos de cultivo, quaisquer das doses aplicadas na superfície elevaram os teores de Ca e Mg mais do que a dose integral de forma incorporada. Esse fato ocorreu porque, com a incorporação, o calcário misturou-secom um mai or vol ume deterra, havendo diluição do seu efeito na camada superficial. Aumentos dos teores de $\mathrm{Ca}$ e $\mathrm{Mg}$ nas camadas superficiais de solos sob SSD devidos à calagem superficial têm sido freqüentemente observados (Sá, 1995; Oliveira \& Pavan, 1996; Caires et al., 1998). Hargrove et al. (1982) e Edwards et al. (1992) relataram maiores teores de Ca e Mg nas camadas superficiais desolos sob SSD do quenaquelas sobSSC.
No sol o com nove anos sob SSD, os teores de Ca e Mg das camadas abaixo de $0-5 \mathrm{~cm}$ não variaram em profundidade, fato que não ocorreu para os demais tempos de cultivo. Contudo, como os teores de Ca e $\mathrm{Mg}$ das camadas inferiores do solo não foram influenciados pelas doses de calcário aplicadas na superfície, este efeito não pode ser atribuído à ação destes tratamentos, mas, sim, a um possível efeito do tempo de cultivo na distribuição desses el ementos em profundidade.

O aumento do tempo de cultivo sob SSD podeter mel horado a fertilidade das camadas inferiores pela possível movimentação física (Sá, 1995; Oliveira \& Pavan, 1996) e química (Sá, 1995; Miyazawa et al., 1996; Caires et al., 1999) dos nutrientes no perfil do solo. Crozier et al. (1999), avaliando 59 locais sob SSD nos Estados Unidos da América do Norte, observaram que as áreas com mais de seis anos de cultivo apresentavam maior homogeneidade dos nutrientes no perfil, não mostrando diferenças entre as camadas de $0-10$ e $10-20 \mathrm{~cm}$ quanto aos valores de $\mathrm{pH}$ e aos teores de Ca. Oliveira \& Pavan (1996) observaram aumento no teor de $\mathrm{Ca}$ e $\mathrm{Mg}$ até $40 \mathrm{~cm}$ de profundidade, após 32 meses da aplicação de cal cário na superfície de um Latossol o argil loso. Em outro Latossolo Vermelho-Escuro textura média, submetido há 15 anos sob SSD, Caires et al. (1999) observaram aumento nos teores de $\mathrm{Ca}$ e $\mathrm{Mg}$ até às camadas de 40-60 e 60-80 cm de profundidade, após 18 meses da aplicação do cal cário na superfície.

No solo com três anos sob SSD, os valores de saturação por bases das camadas superficiais não variaram com as doses de cal cário (Quadro 2). Nas camadas inferiores, no entanto, a incorporação resultou em maior valor, o qual, geralmente, não diferiu dos obtidos com as maiores doses aplicadas na superfície.

No solo com seis anos de cultivo, os valores de saturação por bases na camada de 0-5 am diminuíram quando o cal cário foi incorporado, enquanto a aplicaçãona superfícienãoal terou esta característica.

No sol o com nove anos sob SSD, por sua vez, as mai ores doses de calcário na superfície resultaram em maiores valores de saturação por bases nesta camada. Sá (1995) também observou maiores val ores de saturação por bases nas camadas superficiais de sol os que receberam aplicação superficial de cal cário do que em locais onde o corretivo foi incorporado. No presente trabalho, os maiores valores de saturação por bases na camada superior deveramse aos aumentos dos teores de Ca e Mg nessa camada.

Os teores de nutrientes nas fol has de soja foram pouco alterados com as doses de corretivo, não tendo a concentração de N, P, K eS variado com a cal agem em nenhuma das amostragens (Quadro 3). Estudos de Caires et al. (1998) mostraram que a aplicação de cal cário na superfície não afetou os teores de N, K e S em fol has de soja. Centurion (1988) também 
Quadro 3. Concentração de N, P, K, S, Ca e Mg nas folhas de soja, considerando as doses de calcário, para diferentes tempos de cultivo sob sistema de semeadura direta (TCSSD) e épocas de amostragens

\begin{tabular}{|c|c|c|c|c|c|c|}
\hline \multirow{2}{*}{ TCSSD } & \multicolumn{6}{|c|}{ Dose de calcário(1) } \\
\hline & $100 \mathrm{I}$ & $100 \mathrm{~S}$ & $66,7 \mathrm{~S}$ & $33,3 \mathrm{~S}$ & 0 & Média \\
\hline \multirow{3}{*}{ ano } & \multicolumn{6}{|c|}{$\mathbf{N}$} \\
\hline & \multicolumn{6}{|c|}{$\mathrm{g} \mathrm{kg}^{-1}$} \\
\hline & \multicolumn{6}{|c|}{ Amostragem $1^{(2)}$} \\
\hline \multirow{4}{*}{$\begin{array}{l}\text { Três } \\
\text { Seis } \\
\text { Nove }\end{array}$} & 40,9 & 40,8 & 38,9 & 39,2 & 40,6 & $40,1 b^{(3)}$ \\
\hline & 39,5 & 40,0 & 38,4 & 40,4 & 39,4 & $39,5 \mathrm{~b}$ \\
\hline & 43,6 & 41,8 & 42,4 & 43,9 & 45,0 & $43,4 \mathrm{a}$ \\
\hline & \multicolumn{6}{|c|}{ Amostragem 2} \\
\hline \multirow{6}{*}{$\begin{array}{l}\text { Três } \\
\text { Seis } \\
\text { Nove } \\
\text { C.V. (\%) }\end{array}$} & 38,0 & 36,4 & 37,6 & 35,7 & 35,5 & $36,6 \mathrm{~b}$ \\
\hline & 36,6 & 37,3 & 41,3 & 40,3 & 39,4 & 39,0 a \\
\hline & 39,2 & 37,1 & 37,2 & 39,2 & 40,4 & $38,6 \mathrm{ab}$ \\
\hline & \multirow{2}{*}{\multicolumn{6}{|c|}{$\mathbf{P}$}} \\
\hline & & & & & & \\
\hline & \multicolumn{6}{|c|}{ Amostragem 1} \\
\hline Três & 2,1 & 2,1 & 2,3 & 2,3 & 2,2 & $2,2 a^{(3)}$ \\
\hline Seis & 2,1 & 2,2 & 2,3 & 2,3 & 2,4 & $2,2 \mathrm{a}$ \\
\hline \multirow[t]{2}{*}{ Nove } & 2,2 & 2,2 & 2,3 & 2,3 & 2,4 & $2,3 a$ \\
\hline & \multicolumn{6}{|c|}{ Amostragem 2} \\
\hline \multirow{6}{*}{$\begin{array}{l}\text { Três } \\
\text { Seis } \\
\text { Nove } \\
\text { C.V. (\%) }\end{array}$} & 3,8 & 3,8 & 4,1 & 3,7 & 4,0 & $3,9 a$ \\
\hline & 3,4 & 3,7 & 3,9 & 3,8 & 3,6 & $3,6 \mathrm{~b}$ \\
\hline & 3,3 & 3,3 & 3,1 & 3,7 & 3,4 & $3,3 \mathrm{c}$ \\
\hline & 10,9 & & & & & \\
\hline & & & & & & \\
\hline & & & Am & & & \\
\hline Três & 18,4 & 17,6 & 17,7 & 18,7 & 17,6 & $18,0 a^{(3)}$ \\
\hline Seis & 13,2 & 17,3 & 18,0 & 16,2 & 18,1 & $16,7 \mathrm{a}$ \\
\hline Nove & 15,3 & 16,0 & 15,5 & 18,8 & 18,6 & $16,8 a$ \\
\hline & & & Am & & & \\
\hline Três & 24,1 & 27,9 & 24,8 & 25,7 & 24,9 & $25,5 a b$ \\
\hline Seis & 26,4 & 25,5 & 27,5 & 27,7 & 26,7 & 26,7 a \\
\hline Nove & 22,5 & 22,1 & 21,9 & 25,1 & 22,7 & $23,1 \mathrm{~b}$ \\
\hline C.V. (\%) & 11,5 & & & & & \\
\hline & & & & & & \\
\hline & & & Am & & & \\
\hline Três & 1,5 & 1,4 & 1,4 & 1,4 & 1,5 & $1,4 b^{(3)}$ \\
\hline Seis & 1,5 & 1,6 & 1,7 & 1,6 & 1,8 & $1,6 a$ \\
\hline Nove & 1,9 & 1,6 & 1,5 & 1,6 & 1,7 & $1,7 \mathrm{a}$ \\
\hline & & & $\mathrm{Am}$ & & & \\
\hline Três & 1,5 & 1,5 & 1,4 & 1,5 & 1,5 & $1,5 \mathrm{a}$ \\
\hline Seis & 1,2 & 1,3 & 1,4 & 1,4 & 1,3 & $1,3 \mathrm{~b}$ \\
\hline Nove & 1,3 & 1,3 & 1,3 & 1,5 & 1,4 & $1,4 a b$ \\
\hline C.V. (\%) & 11,8 & & & & & \\
\hline & & & & & & \\
\hline & & & $\mathrm{Am}$ & & & \\
\hline Três & $14,7 \mathrm{abA}^{(3)}$ & 13,1 aA & $14,3 \mathrm{aA}$ & $12,9 \mathrm{aA}$ & $15,0 \mathrm{aA}$ & 14,0 \\
\hline Seis & $16,4 \mathrm{aA}$ & $12,3 \mathrm{aB}$ & $13,0 \mathrm{abB}$ & $13,2 \mathrm{aB}$ & $12,9 \mathrm{abB}$ & 13,6 \\
\hline Nove & $12,7 \mathrm{bA}$ & 11,3 aA & $10,6 \mathrm{bA}$ & 11,9 aA & $11,3 \mathrm{bA}$ & 11,6 \\
\hline & & & Am & & & \\
\hline Três & $7,8 \mathrm{aA}$ & $6,4 \mathrm{aA}$ & $6,5 \mathrm{aA}$ & 6,4 aA & $6,2 \mathrm{aA}$ & 6,7 \\
\hline Seis & 6,9 aA & $7,2 \mathrm{aA}$ & $7,5 \mathrm{aA}$ & $6,9 a A$ & $7,6 \mathrm{aA}$ & 7,1 \\
\hline Nove & $6,6 \mathrm{aA}$ & $6,0 \mathrm{aA}$ & $6,0 \mathrm{aA}$ & $6,1 \mathrm{aA}$ & $6,4 \mathrm{aA}$ & 6,2 \\
\hline C.V. (\%) & 10,2 & & & & & \\
\hline & & & & & & \\
\hline & & & $\mathrm{Am}$ & & & \\
\hline Três & $8,2 \mathrm{aA}^{(4)}$ & $7,5 \mathrm{aA}$ & $8,1 \mathrm{aA}$ & $7,6 \mathrm{aA}$ & $8,1 \mathrm{aA}$ & 7,9 \\
\hline Seis & $8,2 \mathrm{aA}$ & $6,1 \mathrm{bB}$ & $6,2 \mathrm{bB}$ & $6,6 \mathrm{bB}$ & $6,5 \mathrm{bB}$ & 6,7 \\
\hline Nove & $8,3 \mathrm{aA}$ & $7,4 \mathrm{aAB}$ & $6,6 \mathrm{bB}$ & 7,5 abAB & $7,2 \mathrm{abAB}$ & 7,3 \\
\hline & & & $\mathrm{Am}$ & & & \\
\hline Três & $4,4 \mathrm{aA}$ & $3,7 \mathrm{aA}$ & $3,6 \mathrm{aA}$ & $3,4 \mathrm{aA}$ & $3,3 a A$ & 3,7 \\
\hline Seis & 3,3 aA & $3,2 \mathrm{aA}$ & $3,3 \mathrm{aA}$ & 3,4 aA & 3,4 aA & 3,3 \\
\hline Nove & $3,8 \mathrm{aA}$ & $3,9 \mathrm{aA}$ & 3,5 aA & $3,8 \mathrm{aA}$ & 3,9 aA & 3,8 \\
\hline C.V. (\%) & 9,7 & & & & & \\
\hline
\end{tabular}

(1) Percentagem da dose necessária para el evar a saturação por bases a 70\%; I =incorporado a 20 cm; S = aplicado superficialmente. ${ }^{(2)}$ Amostragem 1, realizada no estádio vegetativo da soja (V4) e a 2, no início do florescimento. ${ }^{(3)}$ Letras minúsculas comparam TCSSD dentro de doses em cada amostragem eas maiúsculas comparam as doses dentro de cada TCSSD em cada época de amostragem, pelo teste de Tukey a 5\%. ${ }^{(4)}$ Letras minúsculas comparam TCSSD dentro de cada amostragem, pelo teste de Tukey a $5 \%$. 
observou teores de $\mathrm{N}$ semelhantes em plantas de milho, sob diferentes sistemas de cultivo, mas encontrou maiores teores de $\mathrm{P}$ em fol has de milho cultivadas sob SSD do que nas cultivadas sob SSC. Resultados semel hantes foram encontrados por Muzilli (1983). Caires et al. (1999) não observaram efeito de doses decal cárioaplicadas superficialmente nos teores de $\mathrm{S}$ em folhas de milho, soja e trigo, cultivados em solo com 15 anos sob SSD.

Houve efeito da dose de cal cário nos teores de Ca nas fol has apenas na primeira amostragem no solo com seis anos de cultivo sob SSD (Quadro 3), provavelmente porque esse solo era mais ácido e mais rico em al umíni o que os demais (Quadro 2). Por sua vez, esse efeito apenas na primeira amostragem pode ser atribuído ao fato de o sistema radicular, nessa ocasião, ainda nãoser suficientemente eficiente para explorar o Ca presente no solo. A incorporação do calcário acarretou maior concentração de Ca nas folhas de soja do que a aplicação na superfície, e esta forma deaplicação, em quaisquer das doses, não reduziu os níveis originais de Ca nas fol has.

Caires et al . (1998) não observaram aumento dos teores de Ca em fol has de soja, em função da calagem superficial. Por outrolado, em estudos de Caires et al. (1999), os teores de Ca em fol has de soja aumentaram de forma quadrática com doses de cal cário aplicadas na superfície. Estes (1972) relatou maior concentração de $\mathrm{Ca}$ em plantas de milho cultivadas sob oSSC do que nas cultivadas sob SSD, enquanto Quaggio et al. (1998) constataram que a calagem fez com que o teor de Ca aumentasse em fol has de soja cultivadas sob SSC.

Houve efeito da dose de calcário e do tempo de cultivo nos teores de $\mathrm{Mg}$ nas fol has apenas na primeira amostragem, sobretudo no local com menor tempo de cultivo (Quadro 3). O efeito da calagem apenas na primeira amostragem pode ter a mesma explicação dada para o Ca. No solo com seis anos sob SSD, a incorporação do cal cário resultou no mai or teor de $\mathrm{Mg}$ nas fol has. No solo com nove anos de cultivo, a dose integral, de forma incorporada, possibilitou maior teor do que $2 / 3$ da mesma na superfície. Por outro lado, em todos os tempos de cultivo, a aplicação superficial não modificou os teores em relação à testemunha, mas Caires et al . (1999) observaram aumentos dos teores de Mg nas fol has de soja e trigo, após a aplicação de calcário superficialmente. Estes (1972) encontrou mai or teor deCa e Mg nas plantas de milho cultivadas sob SSC do que nas sob SSD, atribuindo este fato à alta absorção de K pelas plantas sob SSD. No SSC, aumento da concentração de $\mathrm{Mg}$ devido à calagem é comum (Bell, 1996, Oliveira et al., 1997; Quaggio et al., 1998).

As correlações lineares entre os teores de nutrientes nas fol has e no sol o foram sempre baixas e geralmente não-significativas (Quadro 4). As correlações mais importantes foram obtidas entre os teores de $\mathrm{P}$ no solo e nas folhas, no tratamento submetido à aração ( $r=0,59$ a 0,69), eentre os teores de $K$ no solo e nas folhas $(r=0,39$ a 0,52$)$, nos tratamentos sem incorporação. Caires et al. (1999) encontraram correlações positivas e significativas entre os teores de Ca e Mg nas fol has de soja em um solo brasileiro há 15 anos sob SSD.

As produções de grãos de soja e milho não foram influenciadas pelas doses de cal cário (Quadro 5), concordando com os resultados obtidos por Caires et al. (1998) e Caires et al. (1999) em solos ácidos do Paraná, e discordando da recomendação de Raij et al. (1996) de el evar a saturação por bases a 60\% para a soja. Contudo, a recomendação de Raij et al. (1996) de manter a saturação por bases a 50\% para o milho em sol os com matéria orgânica acima de $50 \mathrm{~g} \mathrm{dm}^{-3}$ parece adequada, evidenciando que a dose e o intervalo de aplicação de cal cário em sol os sob SSD devem ser repensados. A razão pela qual as culturas não responderam à calagem pode estar no fato de a saturação por bases inicial (por volta de 50\% na camada de 0-20 cm) já ter sido suficiente. Para Sá

\section{Quadro 4. Coeficientes de correlação linear entre teores de macronutrientes no solo e nas folhas, no tratamento sem incorporação e com incorporação do corretivo, em diferentes épocas e profundidades de coletas}

\begin{tabular}{|c|c|c|c|c|c|}
\hline Profundidade & $\mathbf{P}$ & $\mathbf{K}$ & $\mathrm{Ca}$ & Mg & $\mathbf{S}$ \\
\hline $\mathrm{cm}$ & \multicolumn{5}{|c|}{ Sem incorporação } \\
\hline & \multicolumn{5}{|c|}{ Amostragem $1^{(1)}$} \\
\hline $0-5$ & $-0,05^{\text {ns }}$ & $0,04^{\text {ns }}$ & $0,00^{\text {ns }}$ & $-0,31^{*}$ & $0,00^{\text {ns }}$ \\
\hline $5-10$ & $-0,19^{\text {ns }}$ & $-0,07 n s$ & $0,48^{\text {ns }}$ & $0,49 * *$ & $0,15^{\text {ns }}$ \\
\hline $10-20$ & $0,03^{\text {ns }}$ & $-0,14^{\text {ns }}$ & $0,00^{\text {ns }}$ & $0,41^{* *}$ & $0,01^{\mathrm{ns}}$ \\
\hline \multirow[t]{2}{*}{$20-30$} & $-0,19$ ns & $0,10^{\text {ns }}$ & $-0,34^{*}$ & $0,05^{\text {ns }}$ & $-0,21^{\text {ns }}$ \\
\hline & \multicolumn{5}{|c|}{ Amostragem 2} \\
\hline $0-5$ & $0,27 \mathrm{~ns}$ & $0,47 * *$ & $-0,11^{\text {ns }}$ & $-0,07 \mathrm{~ns}$ & $-0,11^{\mathrm{ns}}$ \\
\hline $5-10$ & $0,26^{\mathrm{ns}}$ & $0,52 * *$ & $-0,13^{\text {ns }}$ & $-0,01^{\text {ns }}$ & $-0,29 *$ \\
\hline $10-20$ & $0,16^{\text {ns }}$ & $0,44 * *$ & $-0,11^{\mathrm{ns}}$ & $0,28^{\text {ns }}$ & $-0,21^{\mathrm{ns}}$ \\
\hline \multirow[t]{3}{*}{$20-30$} & $-0,05^{\text {ns }}$ & $0,39 * *$ & $-0,17^{n s}$ & $0,50 * *$ & $0,30^{*}$ \\
\hline & \multicolumn{5}{|c|}{ Com incorporação } \\
\hline & \multicolumn{5}{|c|}{ Amostragem $1^{(1)}$} \\
\hline $0-5$ & $0,14^{\mathrm{ns}}$ & $-0,69 *$ & $0,43^{\text {ns }}$ & $0,24^{\text {ns }}$ & $0,00^{\text {ns }}$ \\
\hline $5-10$ & $0,25^{\text {ns }}$ & $-0,61^{*}$ & $0,35^{\mathrm{ns}}$ & $0,27^{\mathrm{ns}}$ & $0,07^{\mathrm{ns}}$ \\
\hline $10-20$ & $-0,45^{n s}$ & $-0,48^{\text {ns }}$ & $0,27 \mathrm{~ns}$ & $-0,38^{\text {ns }}$ & $-0,26 \mathrm{~ns}$ \\
\hline \multirow[t]{2}{*}{$20-30$} & $-0,25^{\text {ns }}$ & $-0,56^{\text {ns }}$ & $-0,27^{n s}$ & $-0,10^{\text {ns }}$ & $-0,23^{\text {ns }}$ \\
\hline & \multicolumn{5}{|c|}{ Amostragem 2} \\
\hline $0-5$ & $0,63^{*}$ & $0,42^{\text {ns }}$ & $0,43^{\text {ns }}$ & $0,60 *$ & $0,60^{*}$ \\
\hline $5-10$ & $0,60 *$ & $0,21^{\mathrm{ns}}$ & $0,42^{\text {ns }}$ & $0,61 *$ & $0,61 *$ \\
\hline $10-20$ & $0,69 *$ & $0,56^{\mathrm{ns}}$ & $0,41^{\mathrm{ns}}$ & $0,59 *$ & $0,59 *$ \\
\hline $20-30$ & $0,59 *$ & $0,59 *$ & $-0,23^{\text {ns }}$ & $-0,19^{\text {ns }}$ & $-0,19^{\text {ns }}$ \\
\hline
\end{tabular}

(1) Amostragem 1, realizada no estádio vegetativo da soja (V4) e a 2, no início do florescimento.

$*, * * e^{\text {ns. }}$ Significativos a 5 e 1\% e não-significativo, respectivamente. 
Quadro 5. Produção de soja e milho, considerando a calagem, para diferentes tempos de cultivo sob sistema de semeadura direta (TCSSD)

\begin{tabular}{|c|c|c|c|c|c|c|}
\hline \multirow{2}{*}{ TCSSD } & \multicolumn{6}{|c|}{ Dose de calcário(1) } \\
\hline & 1001 & $100 \mathrm{~S}$ & $66,7 \mathrm{~S}$ & $33,3 \mathrm{~S}$ & 0 & Média \\
\hline \multirow[t]{2}{*}{ ano } & \multicolumn{6}{|c|}{$-\mathrm{kg} \mathrm{ha}^{-1}$} \\
\hline & \multicolumn{6}{|c|}{ Soja (safra 98/99) } \\
\hline $\begin{array}{l}\text { Três } \\
\text { Seis } \\
\text { Nove }\end{array}$ & $\begin{array}{l}3.418 \\
4.153 \\
3.863\end{array}$ & $\begin{array}{l}3.206 \\
4.159 \\
3.453\end{array}$ & $\begin{array}{l}3.198 \\
4.124 \\
3.306\end{array}$ & $\begin{array}{l}3.111 \\
4.384 \\
3.515\end{array}$ & $\begin{array}{l}3.194 \\
4.325 \\
3.724\end{array}$ & $\begin{array}{l}3.225 c^{(2)} \\
4.229 a \\
3.572 b\end{array}$ \\
\hline \multirow[t]{2}{*}{ C.V. (\%) } & 7,0 & & & & & \\
\hline & \multicolumn{6}{|c|}{ Milho (safra 99/00) } \\
\hline $\begin{array}{l}\text { Quatro } \\
\text { Sete } \\
\text { Dez }\end{array}$ & $\begin{array}{l}7.958 \\
8.336 \\
9.300\end{array}$ & $\begin{array}{l}9.244 \\
8.459 \\
9.286\end{array}$ & $\begin{array}{l}9.230 \\
8.695 \\
9.223\end{array}$ & $\begin{array}{l}8.846 \\
8.800 \\
8.597\end{array}$ & $\begin{array}{l}8.535 \\
8.383 \\
8.697\end{array}$ & $\begin{array}{l}8.762 \\
8.535 \\
9.020\end{array}$ \\
\hline C.V. (\%) & 10,6 & & & & & \\
\hline
\end{tabular}

(1) Percentagem da dose necessária para el evar a saturação por bases a $70 \%$; I =incorporado a $20 \mathrm{~cm}$; S = aplicado superficialmente. ${ }^{(2)}$ Letras comparam TCSSD, pelo teste de Tukey a 5\%.

(1995), a calagem em sol os sob SSD nãoé necessária quando a saturação por bases estiver próxima de 45 a 50\%, pel o fato de tal prática provocar deficiências de micronutrientes e, conseqüentemente, diminuir a produção.

A ausência de respostas das cultura à calagem, observada em SSD, pode ser explicada pelo fato de os teores de $\mathrm{Ca}$, M g eK estarem em níveis adequados no solo, apresentando uma relação adequada com o Al trocável (Caires et al., 1998). Por sua vez, Caires et al. (1999) acreditam também que as menores respostas das culturas às doses de cal cário, em SSD, podem estar relacionadas com o menor efeito tóxico do Al, que é complexado pel os compostos orgânicos solúveis presentes nos restos culturais das plantas (K retzchmar et al., 1991; Miyazawa et al., 1996).

O fato de o solo com seis anos de cultivo sob SSD ter apresentado a maior produção de grãos de soja não era esperado. Contudo, as análises de micronutrientes no solo e fol ha (dados não apresentados) mostraram que, neste tempo de cultivo, ocorreram os maiores teores de B e Mn no solo e nas fol has de soja, na primeira amostragem. Também foram observadas correlações positivas entre a produção de grãos e os teores de $B(0,63)$ e de $M n(0,53)$ nas fol has de soja. Assim, os maiores teores destes micronutrientes nas plantas cultivadas no sol o com seis anos sob SSD podem ter influenciado positivamente a produção de grãos, uma vez que reduções de produtividades de soja devidas à deficiência, principalmente de $M n$, têm sido freqüentes quando os valores de $\mathrm{pH}$ são elevados (Novais et al., 1989; Sanzonowic, 1995).

\section{CONCLUSÕES}

1. Com o aumento do tempo de cultivo sob o sistema de semeadura direta, a distribuição de Ca, $\mathrm{Mg}$ e Al e os valores de pH e de saturação por bases no perfil do sol o tornaram-se mais homogêneos.

2. A aplicação da dose total recomendada, ou de 2/3 dela, na superfície do solo, aumentou os teores de Ca e Mg e os valores de saturação por bases na camada de 0-5 cm em todos os tempos de cultivo.

3. Nos solos com os menores tempos de cultivo sob SSD, a incorporação da dose integral de cal cário resultou em maiores valores de $\mathrm{pH}, \mathrm{Ca}, \mathrm{Mg}$ e saturação por bases nas camadas inferiores; nessas camadas, estes atributos não foram alterados pela calagem superficial.

4. Os teores de N, P, K eS nas fol has de soja não variaram com a calagem.

5. O solo com seis anos de cultivo foi o que apresentou a maior produção de soja, enquanto a calagem não influenciou a produtividade de grãos de soja e milho em nenhum tempo de cultivo sob o SSD.

\section{LITE RATURA CITADA}

ARSHAD, M.A.; GILL, K.S.; TURKINGTON, T.K. \& WOODS, D.L. Canola root rot and yield responseto liming and tillage. Agron. J ., 89:17-22, 1997.

BEEL, P.F. Predicting liming needs of soybean using soil $\mathrm{pH}$, aluminum, and manganese soil tests. Comm. Soil Sci. Plant Anal., 27:2749-2764, 1996.

BLEVINS, R.L.; GROVE, J .H. \& KITUR, B.K. Nutrient uptake of corn grown using moldboard plow or no-tillage soil management. Comm. Soil Sci. Plant Anal., 17:401-417, 1986.

CAIRES, E.F.; FONSECA, A.F.; MENDES, J .; CHUEIRI, W.A. \& MADRUGA, E. F. Produção de milho, trigo e soja em função das alterações das características químicas do solo pela aplicação de calcário e gesso na superfície, em sistema de plantio direto. R. Bras. Ci. Solo, 23:315-327, 1999.

CAIRES, E.F.; MADRUGA, E.F.; CHUEIRI, W.A. \& FIGUEIREDO, A. Alterações das características químicas do solo e resposta da soja ao calcário e gesso aplicados na superfície em sistema de cultivo sem preparo do solo. R. Bras. Ci. Solo, 22:27-34, 1998.

CENTURION, J.F. Efeito de sistemas de preparo nas propriedades químicas e físicas de um solo argiloso sob cerrado e na cultura do milho implantada. Piracicaba, Escola Superior de Agricultura “Luiz de Queiroz", 1988. 125p. (Tese de Doutorado)

CROZIER, C.R.; NADERMAN, G.C.; TUCKER, M.R. \& SUGG, R.E. Nutrient and pH stratification with convencional and no-till management. Comm. Soil Sci. Plant Anal., 30:65-74, 1999.

EDWARDS, J .H.; WOOD, C.W.; THURLOW, D.L. \& RUF, M.E. Tillage and crop rotation on fertility status of a Hapludult soil. Soil Sci. Soc. Am. J ., 56:1577-1582, 1992. 
ESTES, O.G. Elemental composition of maize grown under notill and conventional tillage. Agron. J ., 64:733-735, 1972.

FOLLETT, R.F. \& PETERSON, G.A. Surface soil nutrient distribuition as affected by wheatfallow tillage systems. Soil Sci. Soc. Am. J ., 52:141-147, 1988.

FRANZLUEBBERS, A.J . \& HONS, F.M. Soil-profile distribution of primary and secondary plant-available nutrients under conventional and no-tillage. Soil Till. Res.,39:229-239, 1996.

GROVE, J.H. \& BLEVINS, R.L. Correcting soil acidification in continuous corn (Zea mays L.). Comm. Soil Sci. Plant Anal., 19:1331-1342, 1988.

HARGROVE, W.L.; REID, J .T.;TOUCHTON, J .T.\& GALLAHER, R.N. Influence of tillage practices on the fertility status of an acid soil double-cropped to wheat and soybeans. Agron. J ., 74:674-687, 1982.

KITUR, B.K.; PHILLIPS, S.R.; OLSON, K.R. \& EBELHAR, S.A Tillage effects on selected chemical properties of Grantsburg silt Ioam. Comm. Soil Sci. Plant Anal., 25:225-246, 1994.

KRETZSCHMAR, R.M.; HAFNER, H.; BATIONO, A. \& MARSCHNER, H. Long and short-term effects of crop residues on aluminum toxicity, phosphorus availability and growth of pearl millet in an acid sandy soil. Plant Soil, 136:215-223, 1991.

MALAVOLTA, E.; VITTI, G.C. \& OLIVEIRA, S.A. Avaliação do Estado Nutricional das Plantas: princípios eaplicações. 2.ed. Piracicaba, POTAF OS, 1997. 319p.

MIYAZAWA, M.; PAVAN, M.A. \& SANTOS, J .C.F. Effects of addition of crop residues on the leaching of $\mathrm{Ca}$ and $\mathrm{Mg}$ in OXysols. In INTERNATIONAL SYMPOSIUM ON PLANTSOIL INTERACTIONS AT LOW pH 4., Belo Horizonte, 1996. Abstracts. Belo Horizonte, Sociedade Brasileira de Ciência do Solo, 1996. p.8.

MOSCHLER, W.W.; MARTENS, D.C.; RICH, C.I \& SHEAR, G.M. Comparative lime effects on continuous no-tillage and convencional tilled corn. Agron. J ., 65:781-783, 1973.
MUZI LLI, O. I nfluência do sistema de plantio direto, comparado ao convencional, sobre a fertilidade da camada arável do solo. R. Bras. Ci. Solo, 7:95-102, 1983.

NOVAIS, R.F.; NEVES, J .C.L.; BARROS, N.F \& \& SEDIYAMA, T. Deficiência de manganês em plantas de soja cultivadas em solo de cerrado. R. Bras. Ci. Solo, 13:199-204, 1989.

OLIVEIRA, E.L.; PARRA, M.S. \& COSTA, A. Resposta da cultura do milho, em Latossolo Vermel ho-E scuro álico, à calagem. R. Bras. Ci. Solo, 21:65-70, 1997.

OLIVEIRA, E.L. \& PAVAN, M.A. Control of acidity in no-tillage system soybean production. Soil Till. Res.,38:47-57, 1996.

POTTKER, D. \& BEN, J.R. Calagem para uma rotação de culturas no sistema de plantio direto. R. Bras. Ci. Solo, 22:675-684, 1998.

QUAGGIO, J.A.; GALLO, P.B.; FURLANI, A.M.C. \& MASCARENHAS, H.A.A. I soquantas de produtividade de soja e sorgo para níveis de calagem e molibdênio. R. Bras. Ci. Solo, 22:337-344, 1998.

RAIJ , B. van; CANTARELLA, H.; QUAGGIO, J .A. \& FURLANI, A.M.C., eds. Recomendações de calagem e adubação para o Estado deSão Paulo. 2. ed. Campinas, Instituto Agronômico de Campinas, 1996. 285p. (Bol etim técnico, 100)

RAIJ , B. van; QUAGGIO, J .A.; CANTARELLA, H.; FERREIRA, M.E.; LOPES, A.S. \& BATAGLIA, O.C. Análise química do solo para fins de fertilidade. Campinas, Fundação Cargill, 1987. 170p.

SÁ, J .C.M. Calagem em solos sob plantio direto da Região dos Campos Gerais, CentroSul do Paraná. In: SÁ, J .C.M., coord. Curso sobre o manejo do sol o no sistema de plantio direto. Castro, Fundação ABC, 1995. p.73-107.

SANTOS, H.P.; TOMM, G.O. \& LHAMBY, J .C.B. Plantio direto versus convencional: efeito na fertilidade do solo e no rendimento de grãos de culturas em rotação com cevada. R. Bras. Ci. Solo, 19:449-454, 1995.

SANZONOWICZ, C. Deficiência de manganês em solos do cerrado. Piracicaba, POTAFOS, 1995. 7p. (POTAFOS: Informações Agronômicas, 71) 
S. G. MOREIRA et al.

R. Bras. Ci. Solo, 25:71-81, 2001 